\section{Research Square}

\title{
Posterior-only Approach with Unilateral Fenestration for the Treatment of Thoracic and Lumbar Tuberculosis
}

\section{Liyuan Jiang}

Xiangya Hospital Central South University

\section{Zhu Guo}

Xiangya Hospital Central South University

\section{Tianding Wu}

Xiangya Hospital Central South University

Chunyue Duan

Xiangya Hospital Central South University

\section{Yong Cao}

Xiangya Hospital Central South University

Jianzhong Hu ( $\nabla$ jiangliyuan40@126.com )

Xiangya Hospital Central South University https://orcid.org/0000-0001-5903-7172

\section{Research article}

Keywords: Thoracic and lumbar tuberculosis; spinal function units; post-only approach; unilateral fenestration

Posted Date: August 15th, 2019

DOI: https://doi.org/10.21203/rs.2.12962/v1

License: (9) This work is licensed under a Creative Commons Attribution 4.0 International License. Read Full License 


\section{Abstract}

[EXSCINDED] Abstract Abstract Background Unilateral fenestration (UF) is a minimally invasive technique used for the treatment of spinal stenosis. But whether it applies to spinal tuberculosis (TB) is still unknown. We present a retrospective study to evaluate the clinical efficacy and feasibility of a posterioronly approach with unilateral fenestration (PAUF) for treatment of the thoracic and lumbar TB (T

\section{Background}

Spinal tuberculosis (TB) is the most common extrapulmonary TB, accounting for about $50 \%$ of osteoarticular TB [1]. And the thoracic and lumbar segments are the most commonly affected [2]. For spinal TB with severe spinal instability, neurological deficit, and spinal cord compression, surgical treatment is recommended. In recent years, although progress has been made in surgical techniques, surgery for spinal TB remains challenging [3]. Spine surgeons have not reached a consensus on surgical treatment for thoracic and lumbar TB (T\&LTB) [4-6].

To date, there are three main surgical approaches for the treatment of T\&LTB, which are the anterior-only approach (AOA), posterior-only approach (POA), and combined anterior and posterior approach (A\&PA) [7]. As the anterior element destruction is the primary destructive form of spinal TB and the AOA can fully expose the lesion, so most surgeons use an AOA for the treatment of T\&LTB $[1,8]$. However, various scholar reported several disadvantages of it, such as the need for long-time postoperative bed rest, unsatisfying correction of the deformity and challenging procedure at lower lumbar levels [9-11]. The combined A\&PA seems to be a mature surgical method for T\&LTB because it not only supplies a direct vision for debridement but also provide a strong internal fixation. But it always leads to massive surgical trauma and long operation time, which are related to severe complications and prolongation of recovery time [12]. Compare with the AOA and combined A\&PA, the POA cause less surgical trauma and also achieve good clinical outcomes $[7,13,14]$. So the POA is widely accepted by more and more spine surgeon. However, total laminectomy is often required in a POA, which may result in instability of the spine and postoperative back pain $[15,16]$. Hence, an ideal operation still needs to be explored. Unilateral fenestration (UF) is a minimally invasive operation used for the treatment of spinal stenosis. But there were few reports about whether it applies to surgical treatment of T\&LTB. So we performed a study on T\&LTB patient with one or two consecutive functional spinal units involved to evaluate its clinical efficacy and feasibility.

\section{Methods}

\section{General information}

This study protocol was approved by the Ethics Committee of our hospital (201507991). From January. 2007 to December 2016, 83 (45male and 38 female) T\&LTB patients with one or two consecutive spinal functional spinal unit involvement were selected from 355 patients. Patients with any of the following conditions were selected: (1) developing neurological impairment and compression of the spinal cord, (2) 
severe or progressive spinal instability, and (3) poor response to medical management. Exclusion criteria were the following: (1) long-segmental (more than two functional spinal units) involvement with severe destruction of vertebral bodies, (2) extensive anterior abscesses or gravitation abscess, and (3) Patient with rigid kyphoscoliosis; (4) Patient who was found false positive diagnosis after operation and during follow-up. All these 83 patients received the posterior-only approach with unilateral fenestration (POAUF) in our hospital.

The diagnosis of spinal TB was based on clinical manifestations (back pain, lower extremity radiation pain, night sweats, weight loss, and neurological dysfunction), non-specific laboratory findings such as increased erythrocyte sedimentation rate (ESR), elevation of C-reaction protein (CRP), anemia, and hypoproteinemia, radiologic findings (X-ray films, computed tomography, and magnetic resonance imaging), pathological analysis, and positive bacteria culture.

\section{Preoperative preparation}

Every patient routinely received HREZ chemotherapy regimen: isoniazid $5 \mathrm{mg} / \mathrm{kg} /$ day, rifampicin 10 $\mathrm{mg} / \mathrm{kg} /$ day, ethambutol $15 \mathrm{mg} / \mathrm{kg} /$ day, and pyrazinamide $35 \mathrm{mg} / \mathrm{kg} /$ day for 2-4 weeks before the operation. For patients with severe anemia and hypoproteinemia, intensive nutritional treatment was given until patients' anemia, and hypoproteinemia was significantly improved. When progressive neurological deficits appeared during preoperative chemotherapy, we appropriately shortened the time of drug treatment.

\section{Surgical procedure (Figure 2):}

Every patient was placed in the prone position after general anesthesia with tracheal intubation. A median longitudinal incision was made on the skin where the destructive vertebrae located.

Conventionally, we separated paraspinal muscles of destroyed vertebral until the vertebral plate and facet joints exposed. Pedicle screws were inserted into one or two adjacent normal vertebrae above and below destructive vertebrae, and C-arm X-ray was used to indicate the exact location of pedicle screws. A titanium rod was placed on the non-decompression side for temporary stabilization, and then decompression was performed on the other side where damage rang was bigger and paraspinal abscess occurred. Focus on the lesion, 1/3-2/3 of the inferior part of the upper lamina and 1/3-2/3 of the superior part of the lower lamina should be removed, and then ligament flavum was removed by forceps to expose spinal canal. We expanded the spinal canal appropriately according to the scope of the lesion without involving supraspinal and interspinal ligaments. To expand the vision, the operating table was tilted $30^{\circ}$ towards the non-decompression side. We used nerve root retractor to protect the spinal cord and the adjacent upper and lower nerve roots. To prevent the traction of the spinal cord, we cut off the unilateral thoracic nerve root and ligature it if it is necessary. After that, we removed the spinal canal abscess, decompress the spinal cord. Then we opened the destructive intervertebral space, gradually remove the abscess and necrotic tissue, disc, endplate cartilage, sequestrum, and sclerosis bone. We access the paraspinal abscesses via paravertebral sinus and carefully separated abscess wall and sucked out pus and scraped necrotic tissue. Lesion area and sinuses were repeatedly washed with large amounts of 
saline solution, hydrogen peroxide, and diluted isoniazid solution. After decompression and debridement, anti-TB drug ( $1.0 \mathrm{~g}$ of streptomycin powder and $0.2 \mathrm{~g}$ of isoniazid) was put into the lesion area.

Allogeneic bone with appropriate length and size was grafted in intervertebral space after mixing with streptomycin powder. The defect area of the vertebral plate resulted from decompression, was repaired by an allogeneic bone plate that was trimmed to fit shape. Then, the rod on the decompression side and cross-linkage (if necessary) were connected to immobilize the grafted bone plate. For patients with kyphosis deformity, the bone graft should be performed after the correction of kyphosis. A drainage tube was placed after all the above operation.

\section{Postoperative treatment}

Antibiotic was regularly used during the operation and within three days post-operatively. After the amount of the fluid drainage reduced to $20 \mathrm{ml}$ per 24 hours, we removed the drainage tube. Bracing protection was recommended to last 3 months post-operation. All patients received oral HREZ chemotherapy postoperatively. Six months later, pyrazinamide was discontinued. Then, patients received nine to 12-month regimens of HRE chemotherapy (6HREZ/9-12HRE). For some patients with neurological damage, early rehabilitation treatment was allowed to improve neurological function.

\section{Follow-up index}

All patients were examined clinically and radiologically at 3, 6, and 12 months after surgery, and then once a year after that. Blood loss and operative time were recorded during the procedure. The following indexes were recorded preoperatively, postoperatively and during follow-up: (1) Cobb angle; (2) neurologic status by ASIA classification; (3) VAS score of back pain; (4) blood indicators such as ESR, CRP, and hepatorenal function; and (5) complications and adverse events.

\section{Statistical analysis}

SPSS 19.0 software (SPSS, Inc., Chicago, IL, USA) was used for statistical analysis. Kyphosis angle, VAS, ESR, and CRP were statistically analyzed by paired t-test. Neurological function was statistically analyzed by the Wilcoxon signed rank test. The discrepancy of normal distribution was analyzed by the rank-sum test with a significance level of 0.05 .

\section{Results}

\section{Population characteristics and surgical conditions (Table 1, Figure 1)}

Among these 83 patients, there were 45 male and 38 female with average age $47.4 \pm 15.9$ (17-79) year old. The mean follow-up time was $46.9 \pm 13.1$ (24-72) months. The diseased segments are presented in Figure 1. The average of operation time and blood loss volumes were 156.6 \pm 19.9 minutes (120-230 minutes) and $471.8 \pm 81.4 \mathrm{ml}(180-650 \mathrm{ml})$.

\section{Clinical outcomes (Table 2 and table 3 )}


The Mean kyphotic Cobb angle significantly reduced from preoperative $23.0^{\circ} \pm 15.3^{\circ}$ to postoperative $8.3^{\circ}$ $\pm 11.0^{\circ}$ and the mean correction of kyphotic Cobb angle was $14.8 \pm 5.5^{\circ}$ (p $₫ 0.001$ ). At the last follow-up, the mean postoperative kyphotic angle was $9.9^{\circ} \pm 11.4^{\circ}$ and the average loss angle was $1.70 \pm 1.07$. There were 38 patients with neurological impairments before surgery (four cases in grade $B, 13$ case in grade $C$ and 21 cases in grade D), and at last follow-up point (at least two years after surgery), 35 patients showed a neurological function improvement (two case improved from $B$ to $C, 11$ cases improved from $C$ to $D$, two case improved from $C$ to $E, 20$ cases improved from $D$ to $E$ ). And the rate of neurological improvement was $89.2 \%$. The VAS pain score significantly decreased from preoperative $6.9 \pm 1.1$ to $1.3 \pm 0.7$ one year post operation ( $\mathrm{p} \otimes 0.001$ ). The average preoperative ESR and CRP was $43.8 \pm 16.7 \mathrm{~mm} / \mathrm{h}$ and $40.5 \pm 17.2 \mathrm{mg} / \mathrm{L}$, which returned to normal level at the six months after surgery. X-ray and CT were used to assess bone fusion, and all patients achieved definitive fusion within 13 (mean fusion time is 8.1 \pm 1.2 months) months after surgery according to the modified criteria of Lee et al.[17] (Figure 3, 4).

\section{Complications and adverse events (Table 4)}

After the operation, cerebrospinal fluid leakage occurred in two patients, superficial wound infections occurred in three cases, mild intestinal obstruction happened in 11 cases, and deep vein thrombosis of lower limb happened in one patient. During the follow-up, three patients suffered no neurological function improvement, two patients suffered intercostal neuralgia, and three patients suffered impairment of liver

$\&$ kidney's function. All these complications were cured and relieved effectively. There were three cases of recurrent, who were cured after the second debridement and adjustment of chemotherapy regimens.

\section{Discussion}

\section{Surgery is an auxiliary treatment for spinal TB}

Spinal TB is a local infectious disease caused by Mycobacterium tuberculosis. According to different pathological status, specific treatment such as rest, motion-suppression, intensive nutrition, anti-TB drugs and surgical treatment should be taken. Among these, medications currently remain the essential method for the treatment of spinal TB. Majority of spinal TB cases can be cured by conventional pharmacotherapy [18]. Studies have demonstrated that it was difficult for anti-TB drugs to fully penetrate into the center of the lesion in some spinal TB patients due to the 'barrier', which was regarded as the sclerotic bone around the lesion $[19,20]$. However, the sclerotic bone around the focal can be removed by surgery, allowing penetration of anti-TB drugs to kill the Mycobacterium tuberculosis. Anti-TB drugs should be used during the whole treatment for spinal TB, as it is fundamentally effective against Mycobacterium tuberculosis [21]. Surgery, an auxiliary treatment for spinal TB, plays a more critical role in debridement, deformity correction and reconstruction of spinal stability.

The reasons we choose POAUF to treat T\&LTB 
According to three-column spine theory [22], the spine is divided into three columns: the anterior column, middle column, and posterior column. Spinal TB predominantly damages the anterior column [23]. When the anterior column is in a pathological state, the original spine biomechanical properties change significantly, resulting in spinal load redistribution after attachment. In a pathological state or a fixed state, the function of the anterior column completely diminishes. Thus, the posterior column bears all the pressure, indicating that the posterior structure of the spine plays a very assignable role in maintaining the spinal stability [24]. However, although primary POA with total laminectomy is regarded as an effective treatment for T\&LTB, it always results in posterior column destruction which will further jeopardize the stability of the spine and result in many complications, such as iatrogenic instability, epidural scar adhesion, and lumbar failure syndrome [25]. So less invasive technique still needs to explore.

The UF for the treatment for spinal stenosis was initially described in 1998 by Mackay DC et al. [26]. And then, more and more spine surgeons got success in treating spinal stenosis by using UF $[27,28]$.

Biomechanics experiments have proved that the stability of UF is superior to that of bilateral fenestration and laminectomy [29]. Moreover, some spine surgeons achieved bilateral decompression using UF [30]. Most recently, various authors also have recommended posterior surgical techniques that preserve posterior midline structures [31,32]. So to minimize the surgical trauma to the posterior column, we used POAUF to treat T\&LTB with one or two consecutive function spinal unit involved.

\section{Why POAUF is effective for the treatment for T\&LTB}

In our operation, we resected the unilateral partial lamina to do debridement and decompression. The spinous process, interspinous ligament, supraspinal ligament, facet joint, and most of the lamina had been reserved. But, reducing the extent of laminectomy means narrowing the surgical vision. It brings a big challenge for decompression and debridement. However, according to our experience, what causes the compression of the spinal cord is abscess and loose necrotic tissue, which can be easily cleared using aspirator and curette through UF. So decompression of spinal cord was smoothly achieved in our procedure. After decompression, we also cleared out the residue pus, necrotic tissue, sequestrum, and sclerosis bone at the intervertebral space. To remove the lesion on the non-decompression side, we tilted the operating table with $30^{\circ}$ towards the non-decompression side, and then we can see the contralateral lesion and clear it. The removal of sclerosis bone plays a very critical role in the effective debridement. Because sclerosis bone is regarded as the "wall" prevent the anti-TB drugs seep into the lesion area [19, 20,33]. To ensure the sclerosis bone has been removed, we kept curetting the sclerosis bone out until we can see the normal cancellous bone and blood flow out from the marrow cavity. After removal of sclerosis bone, TB bacilli at the focal would be killed by the medication. And after at least a two-year follow-up, our operation achieved good clinical outcomes. Although we may not be able to do extensive anterior debridement with UF, it did not influence the effectiveness of the POAUF. Wang et al. also got a good clinical out without anterior debridement in their previous study [34]. So, our data showed that extensive anterior debridement might not necessary in the treatment of T\&LTB. 


\section{Advantages and limitation of POAUF}

Kumar et al. [35] and Liu et al. [14] also described using posterior surgery alone in patients with active thoracolumbar TB. All patients underwent hemi- or total laminectomy for removal of the lesion. They also achieved good clinical outcomes in their study. But in their operation, the blood loss was both over $600 \mathrm{ml}$, which was significantly more than that in our operation. And at last follow-up time point, the mean loss of correction was $4.3^{\circ}$ and $3.2^{\circ}$ in their study, which was more than $1.70^{\circ}$ in our study. According to these data, our operation seems to cause less surgical trauma and maintain a better correction of kyphosis. In addition, our operation also has these advantages below: 1) minimize the surgical damage to the posterior column; 2) causing small interference to the spinal cord when doing decompression and bone graft; 3) surgery has little impact to organs in the chest and abdominal cavity. Although there were many advantages of POAUF, it also has its limitations. We don't recommend our operation for patients with extensive paraspinal abscess, multiple vertebral involvement, and rigid kyphoscoliosis.

To sum up, surgery plays a supporting role in the treatment of spinal TB. Extensive resection of the posterior column and excessive debridement is not necessary and recommended. Posterior minimally invasive surgery, which can reserve the midline structure is a good choice for T\&LTB.

\section{Conclusion}

Posterior-only approach with unilateral fenestration is not only an effective and feasible surgical treatment for thoracic and lumbar spinal TB with one or two consecutive functional spinal units involved but also a minimally invasive technique that can minimize the surgical damage to the posterior column.

\section{Abbreviations}

TB: tuberculosis; UF: Unilateral fenestration; POAUF: posterior-only approach with unilateral fenestration; $\mathrm{T}<\mathrm{B}$ : thoracic and lumbar TB; anterior-only approach (AOA); posterior-only approach (POA); combined anterior and posterior approach (A\&PA); HREZ: isoniazid, rifampin, ethambutol, and pyrazinamide; HRE: isoniazid, rifampin, and ethambutol; ESR: Erythrocyte sedimentation rate; CRP: C-reaction protein; CT: Computed tomography; MRI: Magnetic resonance imaging; ASIA classification $₫$ American Spinal Injury Association classification; VAS: Visual Analogue Scale scores of pain

\section{Declarations}

\section{Ethics approval and consent to participate}

Written informed consent was obtained from all patients. This study protocol was approved by the Ethics Committee of Xiangya Hospital. And the authorization number is: 201507991

\section{Consent to publish}


All authors agree to publish in BMC MUSCULOSKELETAL DISORDERS.

\section{Availability of data and materials}

All datasets on which the conclusions of the manuscript rely were presented in the main paper. The detail data is in medical record data base of our hospital.

\section{Competing interests}

The authors declare that they have no competing interests.

\section{Funding}

This research was supported by the National Natural Science Foundation of China (Nos 81874004, 81672174)

\section{Competing interests}

The authors declare that they have no competing interests.

\section{Author's contributions}

Jianzhong Hu did the surgery for patients. Jianzhong Hu, Yong Cao and Liyuan Jiang participated in the design of this study. Liyuan Jiang drafted the Manuscript with the help from Yong Cao. Zhu Guo, Tianding Wu and Chunyue Duan helped Liyuan Jiang collected clinical data and important background information. All authors read and approved the final manuscript.

\section{Acknowledgment}

This work was partly supported by the Fundamental Research Funds for the Central Universities of Central South University (No.2018zzts254).

\section{References}

1. Jain AK, Dhammi IK: Tuberculosis of the spine: a review. Clin Orthop Relat Res 2007, 460:39-49.

2. Shi T, Zhang Z, Dai F, Zhou Q, He Q, Luo F, Hou T, Xu J: Retrospective Study of 967 Patients With Spinal Tuberculosis. ORTHOPEDICS 2016, 39(5):e838-e843.

3. Rasouli MR, Mirkoohi M, Vaccaro AR, Yarandi KK, Rahimi-Movaghar V: Spinal tuberculosis: diagnosis and management. Asian Spine J 2012, 6(4):294-308.

4. Cui X, Ma YZ, Chen X, Cai XJ, Li HW, Bai YB: Outcomes of different surgical procedures in the treatment of spinal tuberculosis in adults. Med Princ Pract 2013, 22(4):346-350.

5. Ekinci S, Agilli M, Ersen O, Ekinci GH: Surgical treatment of tuberculous spondylodiscitis. Eur Rev Med Pharmacol Sci 2015, 19(5):700-701. 
6. Li L, Xu J, Ma Y, Tang D, Chen Y, Luo F, Li D, Hou T, Zhou Q, Dai F et al: Surgical strategy and management outcomes for adjacent multisegmental spinal tuberculosis: a retrospective study of forty-eight patients. Spine (Phila Pa 1976) 2014, 39(1):E40-E48.

7. Wang LJ, Zhang HQ, Tang MX, Gao QL, Zhou ZH, Yin XH: Comparison of Three Surgical Approaches for Thoracic Spinal Tuberculosis in Adult: Minimum 5-Year Follow Up. Spine (Phila Pa 1976) 2017, 42(11):808-817.

8. Watts HG, Lifeso RM: Tuberculosis of bones and joints. J BONE JOINT SURG AM 1996, 78(2):288298.

9. Ha KY, Shin JH, Kim KW, Na KH: The fate of anterior autogenous bone graft after anterior radical surgery with or without posterior instrumentation in the treatment of pyogenic lumbar spondylodiscitis. Spine (Phila Pa 1976) 2007, 32(17):1856-1864.

10. Soares DBJ, Batista N, Tirado A, Fernandes P: [Surgical treatment of spinal tuberculosis: an orthopedic service experience]. Acta Med Port 2013, 26(4):349-356.

11. Ha KY, Shin JH, Kim KW, Na KH: The fate of anterior autogenous bone graft after anterior radical surgery with or without posterior instrumentation in the treatment of pyogenic lumbar spondylodiscitis. Spine (Phila Pa 1976) 2007, 32(17):1856-1864.

12. Memtsoudis SG, Vougioukas VI, Ma Y, Gaber-Baylis LK, Girardi FP: Perioperative morbidity and mortality after anterior, posterior, and anterior/posterior spine fusion surgery. Spine (Phila Pa 1976) 2011, 36(22):1867-1877.

13. Wang ST, Ma HL, Lin CP, Chou PH, Liu CL, Yu WK, Chang MC: Anterior debridement may not be necessary in the treatment of tuberculous spondylitis of the thoracic and lumbar spine in adults: a retrospective study. BONE JOINT J 2016, 98-B(6):834-839.

14. Liu Z, Zhang P, Zeng H, Xu Z, Wang X: A comparative study of single-stage transpedicular debridement, fusion, and posterior long-segment versus short-segment fixation for the treatment of thoracolumbar spinal tuberculosis in adults: minimum five year follow-up outcomes. INT ORTHOP 2018, 42(8):1883-1890.

15. Tai CL, Hsieh PH, Chen WP, Chen LH, Chen WJ, Lai PL: Biomechanical comparison of lumbar spine instability between laminectomy and bilateral laminotomy for spinal stenosis syndrome - an experimental study in porcine model. BMC Musculoskelet Disord 2008, 9:84.

16. Datta G, Gnanalingham KK, Peterson D, Mendoza N, O'Neill K, Van Dellen J, McGregor A, Hughes SP: Back pain and disability after lumbar laminectomy: is there a relationship to muscle retraction? NEUROSURGERY 2004, 54(6):1413-1420, 1420.

17. Lee JS, Moon KP, Kim SJ, Suh KT: Posterior lumbar interbody fusion and posterior instrumentation in the surgical management of lumbar tuberculous spondylitis. J Bone Joint Surg Br 2007, 89(2):210214.

18. A 15-year assessment of controlled trials of the management of tuberculosis of the spine in Korea and Hong Kong. Thirteenth Report of the Medical Research Council Working Party on Tuberculosis of the Spine. J Bone Joint Surg Br 1998, 80(3):456-462. 
19. Ge Z, Wang Z, Wei M: Measurement of the concentration of three antituberculosis drugs in the focus of spinal tuberculosis. EUR SPINE J 2008, 17(11):1482-1487.

20. Liu $P, Z$ hu $Q$, Jiang J: Distribution of three antituberculous drugs and their metabolites in different parts of pathological vertebrae with spinal tuberculosis. Spine (Phila Pa 1976) 2011, 36(20):E1290E1295.

21. Mukherjee JS, Rich ML, Socci AR, Joseph JK, Viru FA, Shin SS, Furin JJ, Becerra MC, Barry DJ, Kim JY et al: Programmes and principles in treatment of multidrug-resistant tuberculosis. LANCET 2004, 363(9407):474-481.

22. Denis $F$ : The three column spine and its significance in the classification of acute thoracolumbar spinal injuries. Spine (Phila Pa 1976) 1983, 8(8):817-831.

23. Garg RK, Somvanshi DS: Spinal tuberculosis: a review. J SPINAL CORD MED 2011, 34(5):440-454.

24. Hernandez-Labrado GR, Polo JL, Lopez-Dolado E, Collazos-Castro JE: Spinal cord direct current stimulation: finite element analysis of the electric field and current density. MED BIOL ENG COMPUT 2011, 49(4):417-429.

25. Pietrantonio A, Trungu S, Fama I, Forcato S, Miscusi M, Raco A: Long-term clinical outcomes after bilateral laminotomy or total laminectomy for lumbar spinal stenosis: a single-institution experience. NEUROSURG FOCUS 2019, 46(5):E2.

26. Mackay DC, Wheelwright EF: Unilateral fenestration in the treatment of lumbar spinal stenosis. $\mathrm{Br} J$ Neurosurg 1998, 12(6):556-558.

27. Xu B, Xu H, Ma X, Liu Y, Yang Q, Jiang H, Li N, Ji N: Bilateral decompression and intervertebral fusion via unilateral fenestration for complex lumbar spinal stenosis with a mobile microendoscopic technique. Medicine (Baltimore) 2018, 97(4):e9715.

28. Xu BS, Tan QS, Xia Q, Ji N, Hu YC: Bilateral decompression via unilateral fenestration using mobile microendoscopic discectomy technique for lumbar spinal stenosis. ORTHOP SURG 2010, 2(2):106110.

29. Hamasaki T, Tanaka N, Kim J, Okada M, Ochi M, Hutton WC: Biomechanical assessment of minimally invasive decompression for lumbar spinal canal stenosis: a cadaver study. J SPINAL DISORD TECH 2009, 22(7):486-491.

30. Liao Y, Ye R, Tang Q, Tang C, Ma F, Luo N, Wang G, Wang Q, Zhong D: [Application of ultrasonic osteotome in the posterior lumbar interbody fusion surgery by unilateral fenestration and bilateral decompression in the treatment of degenerative lumbar spinal stenosis]. Zhongguo Xiu Fu Chong Jian Wai Ke Za Zhi 2019, 33(4):416-422.

31. Celik SE, Celik S, Goksu K, Kara A, Ince I: Microdecompressive laminatomy with a 5-year follow-up period for severe lumbar spinal stenosis. J SPINAL DISORD TECH 2010, 23(4):229-235.

32. Thome C, Zevgaridis D, Leheta O, Bazner H, Pockler-Schoniger C, Wohrle J, Schmiedek P: Outcome after less-invasive decompression of lumbar spinal stenosis: a randomized comparison of unilateral laminotomy, bilateral laminotomy, and laminectomy. J Neurosurg Spine 2005, 3(2):129-141. 
33. Jin W, Wang Q, Wang Z, Geng G: Complete debridement for treatment of thoracolumbar spinal tuberculosis: a clinical curative effect observation. SPINE J 2014, 14(6):964-970.

34. Wang ST, Ma HL, Lin CP, Chou PH, Liu CL, Yu WK, Chang MC: Anterior debridement may not be necessary in the treatment of tuberculous spondylitis of the thoracic and lumbar spine in adults: a retrospective study. BONE JOINT J 2016, 98-B(6):834-839.

35. Kumar MN, Joseph B, Manur R: Isolated posterior instrumentation for selected cases of thoracolumbar spinal tuberculosis without anterior instrumentation and without anterior or posterior bone grafting. EUR SPINE J 2013, 22(3):624-632.

\section{Tables}

\section{Table 1}

General data of patients

\begin{tabular}{lllll}
\hline Gender & Age (years) & follow-up time(mon $\square$ & Operation time (min) & Blood loss (ml) \\
\hline $\begin{array}{l}\text { Male Female } \\
45 \quad 38\end{array}$ & $47.4 \pm 15.9$ & $46.9 \pm 13.1$ & $156.2 \pm 19.9$ & $471.8 \pm 81.4$ \\
\hline
\end{tabular}

\section{Table 2}

Clinical outcomes 1

\begin{tabular}{lccccccl}
\hline Schedule & \multicolumn{4}{c}{ ASIA classification } & ESR(mm/h) & CRP (mg/L) \\
\hline & A & B & C & D & E & & \\
Pre-op & & 4 & 13 & 21 & 45 & $43.8 \pm 16.7$ & $40.5 \pm 17.2$ \\
LFU & & 2 & 2 & 14 & 65 & $10.3 \pm 3.6$ & $7.2 \pm 2$. \\
\hline
\end{tabular}

Pre-op: pre-operation; LFU: last follow-up point; ESR: Erythrocyte Sedimentation Rate; CRP: Creactive protein; ASIA classification: American spinal cord association classification.

Neurological function by ASIA classification, Wilcoxon signed rank test was used to comparing the grade of neurological function, there was a significant difference between pre-operation and post-operation $(\mathrm{P}<0.001)$

\section{Table 3}

Clinical outcomes 2 


\begin{tabular}{ll}
\hline Schedule & results \\
\hline VAS score & \\
Pre-op & $6.9 \pm 1.1$ \\
TMP & $2.7 \pm 0.9$ \\
LFU & $1.3 \pm 0.7$ \\
Kyphosis angle $\left(^{\circ}\right)$ & $23.0 \pm 15.3$ \\
Pre-op & $8.3 \pm .11 .0$ \\
Post-op & $9.9 \pm 11.4$ \\
LFU & $8.1 \pm 1.2$ \\
Bone fusion time (Mon) & \\
\hline
\end{tabular}

Pre-op: pre-operation; Post-op: Post-operation; TMP: three months post operation; LFU: last follow-up point; VAS: Visual Analogue Scale scores of pain; Kyphosis angle and VAS score were analyzed by paired t-test, there was a significant difference between pre-operation and postoperation $(\mathrm{P}<0.001)$.

\section{Table 4}

Complications and adverse events

\begin{tabular}{ll}
\hline Complications and adverse events & \\
\hline Cerebrospinal fluid leakage & 2 \\
Superficial wound infections & 3 \\
Mild intestinal obstruction & 11 \\
Deep Vein Thrombosis of lower limb & 1 \\
No neurological improvement & 3 \\
Recurrence & 3 \\
Impairment of liver \& Kidney' s function & 3 \\
Intercostal neuralgia & 2 \\
Total & 28 \\
\hline
\end{tabular}

Figures 


\section{2}

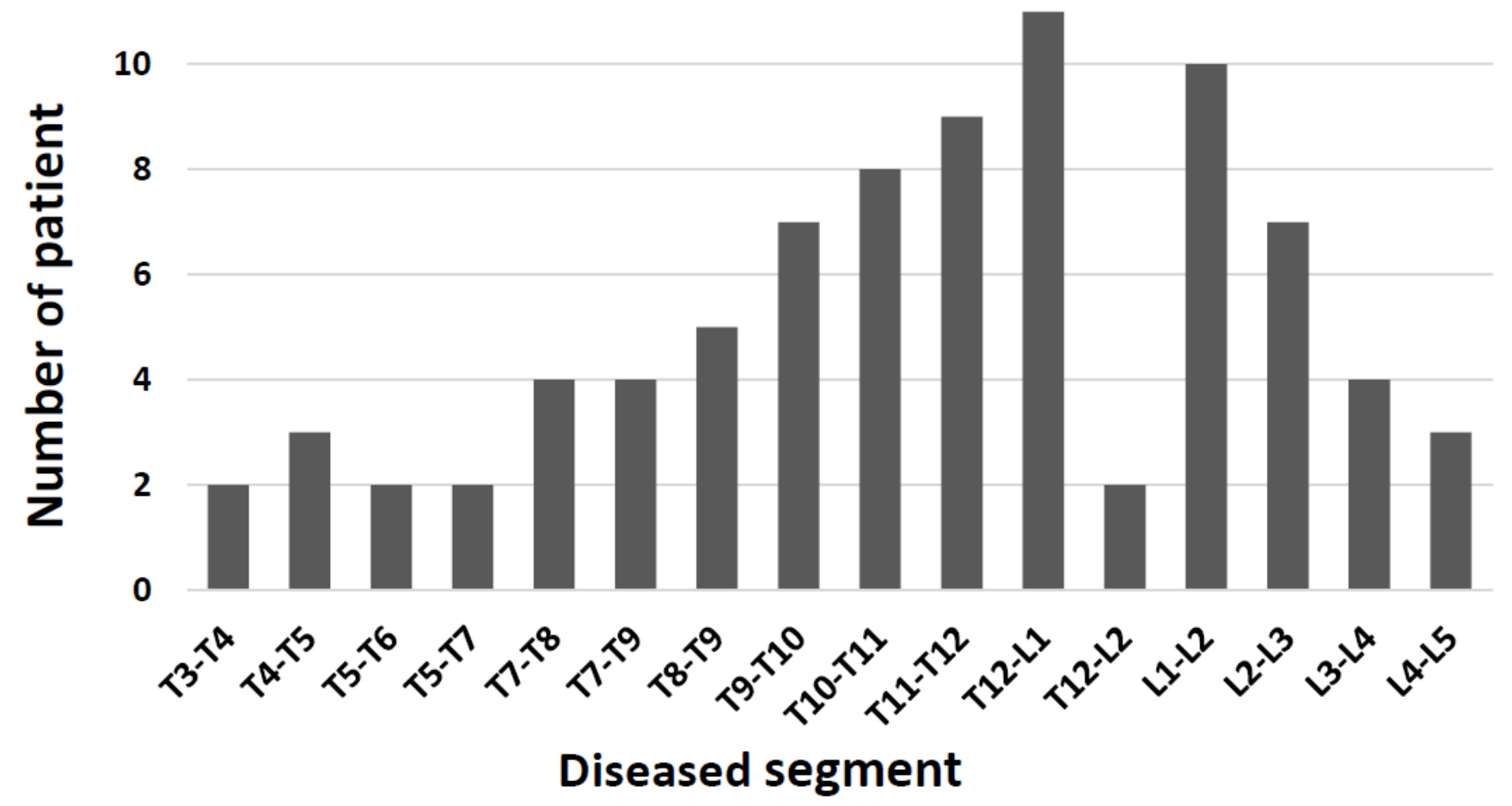

Figure 1

The distribution of involved thoracic and thoracolumbar vertebras 

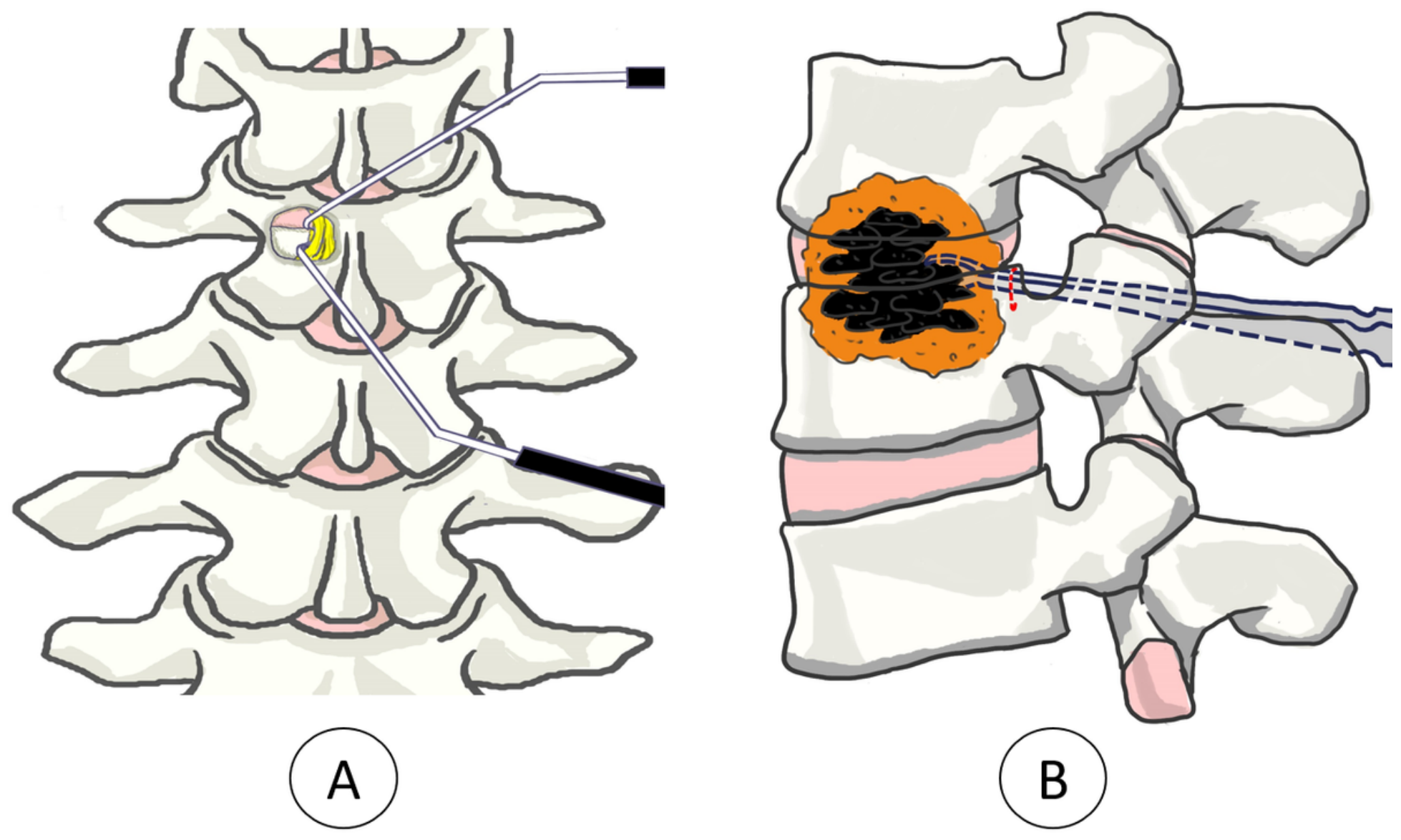

Figure 2

The scope of resection. (A) Posterior approach with unilateral fenestration; (B) debridement via intervertebral space in the treatment of spinal tuberculosis. 

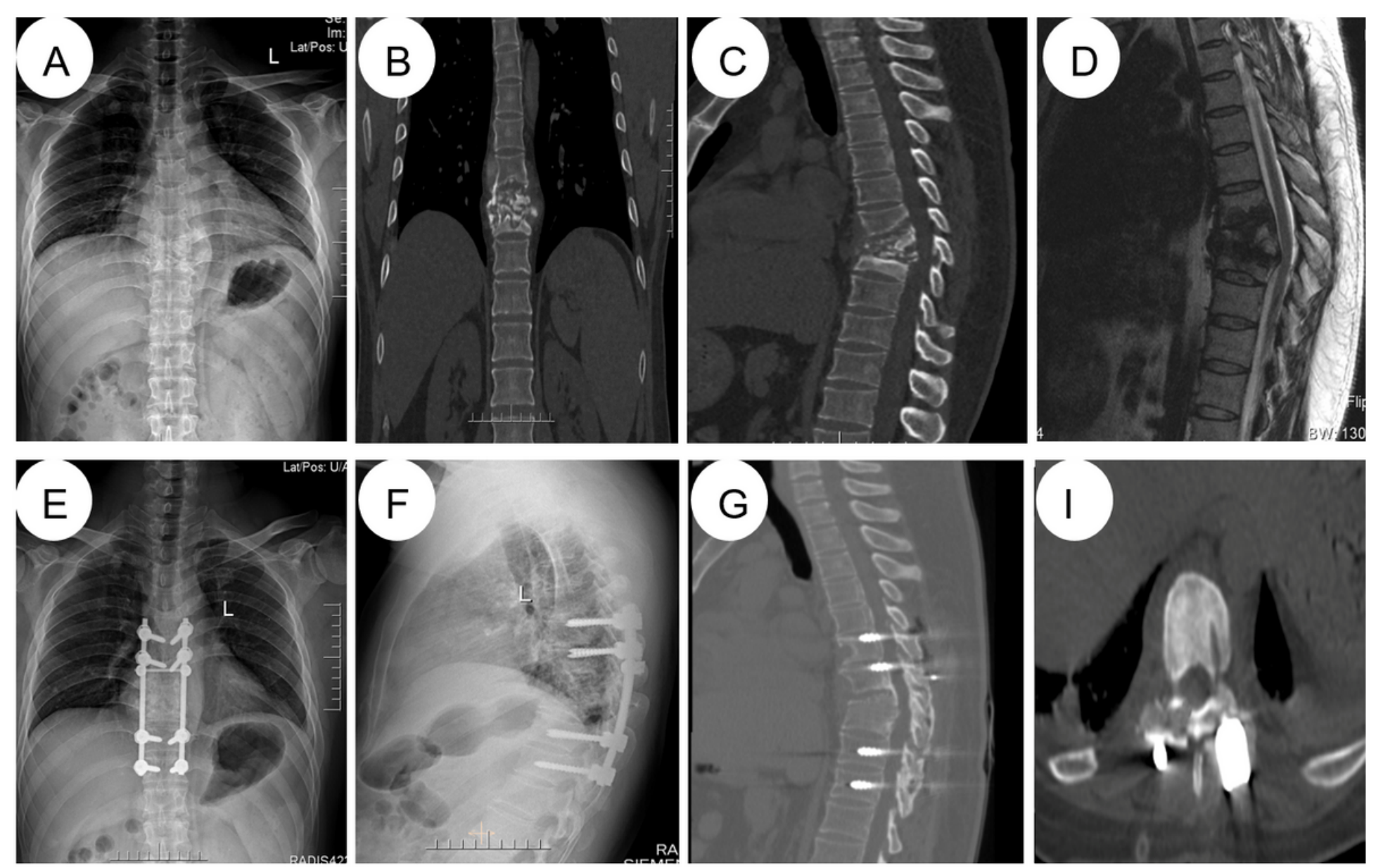

Figure 3

A 53-year-old male with T7-T9 lesions Figure A-D are pre-operative images ( $\mathrm{X}$-ray, CT and MRI) show destruction of T7-T9 vertebral body with moderate kyphosis deformity and compression of spinal cord. Figure $\mathrm{E}, \mathrm{F}$ are postoperative $\mathrm{X}$-ray images indicating the correction of kyphosis by posterior fixation. Sagittal CT-scan (G) shows satisfied bone fusion. Figure I shows the scope of resection. 

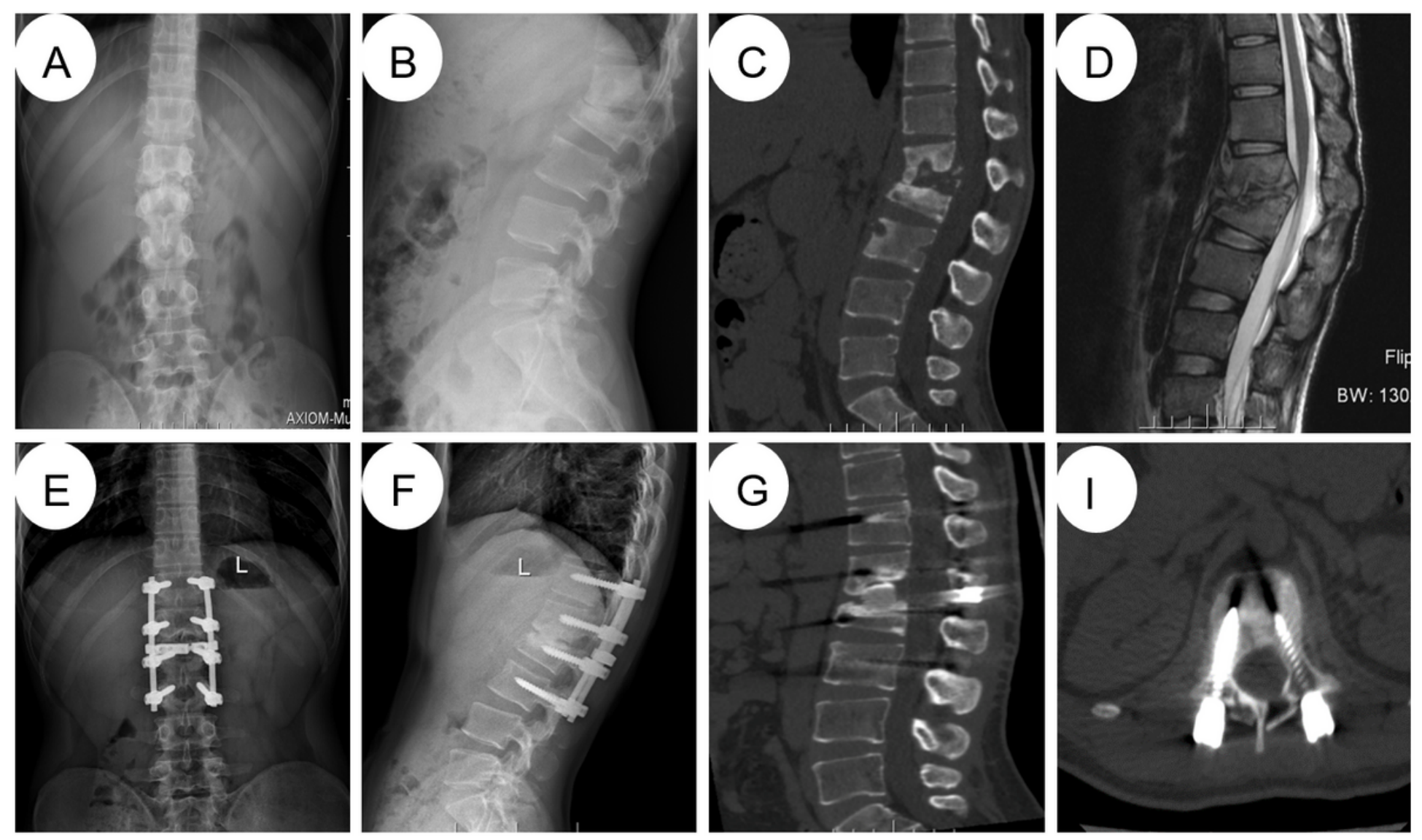

\section{Figure 4}

A 26-year-old male with L1-L2 lesions Figure A-D are pre-operative images ( $\mathrm{X}$-ray, CT, and MRI) show the destruction of L1-L2 vertebral with moderate kyphosis deformity. Figure E, F are postoperative X-ray images indicating the correction of kyphosis by posterior fixation. Sagittal CT-scan (G) shows satisfied bone fusion. Figure I shows the scope of resection. 